\begin{tabular}{|c|l|}
\hline Title & Quasiparticle properties of a single al pha particle in cold neutron matter \\
\hline Author(s) & Nakano, Eiji; Iida, Kei; Horiuchi, Wataru \\
\hline Citation & $\begin{array}{l}\text { Physical Review C, 102(5), 055802 } \\
\text { https:/doi.org/10.1103/PhysRevC.102.055802 }\end{array}$ \\
\hline Issue Date & 2020-11-02 \\
\hline Doc URL & http://hdl.handle.net/2115/80013 \\
\hline Rights & @2020 A merican Physical Society \\
\hline Type & article \\
\hline File Information & PhysRevC.102-5_055802.pdf \\
\hline
\end{tabular}

Instructions for use 


\title{
Quasiparticle properties of a single $\alpha$ particle in cold neutron matter
}

\author{
Eiji Nakano* \\ Department of Mathematics and Physics, Kochi University, Kochi 780-8520, Japan \\ and Institut für Theoretische Physik, Goethe Universität Frankfurt, D-60438 Frankfurt am Main, Germany
}

Kei Iida ${ }^{\dagger}$

Department of Mathematics and Physics, Kochi University, Kochi 780-8520, Japan

Wataru Horiuchi ${ }^{\ddagger}$

Department of Physics, Hokkaido University, Sapporo 060-0810, Japan

(Received 16 June 2020; revised 18 September 2020; accepted 14 October 2020; published 2 November 2020)

\begin{abstract}
Light clusters such as $\alpha$ particles and deuterons are predicted to occur in hot nuclear matter as encountered in intermediate-energy heavy-ion collisions and protoneutron stars. To examine the in-medium properties of such light clusters, we consider a much simplified system in which, like an impurity, a single $\alpha$ particle is embedded in a zero-temperature, dilute gas of noninteracting neutrons. By adopting a non-self-consistent ladder approximation for the effective interaction between the impurity and the gas, which is often used for analyses of Fermi polarons in a gas of ultracold atoms, we calculate the quasiparticle properties of the impurity, i.e., the energy shift, effective mass, quasiparticle residue, and damping rate.
\end{abstract}

DOI: 10.1103/PhysRevC.102.055802

\section{INTRODUCTION}

Understanding the properties of hot nuclear matter as encountered in supernova cores is essential in describing various phenomena such as neutrino bursts, nucleosynthesis, and formation of neutron stars [1]. Remarkably, this matter can be opaque even to neutrinos, which in turn play a role in carrying a released gravitational energy of order $10^{53} \mathrm{erg}$ during stellar collapse by diffusing out of supernova cores and at the same time in depositing a sufficient energy onto the material to cause a supernova explosion at which the total kinetic energy is of order $10^{51} \mathrm{erg}$. Here, it is significant to note that at finite temperature, a nonzero number of light clusters such as $\alpha$ particles, deuterons, tritons, and ${ }^{3} \mathrm{He}$ nuclei appear even in chemical equilibrium. These clusters, if sufficiently present in supernova cores, can play a role in scattering or absorbing the outgoing neutrinos. For example, even an ${ }^{56} \mathrm{Fe}$ nucleus, one of the most stable nuclear configurations in vacuum, can decompose into $13 \alpha$ particles and four neutrons by absorbing a $\gamma$-ray photon of energy in excess of $-Q=124 \mathrm{MeV}$. If an ${ }^{56} \mathrm{Fe}$ nucleus is simply assumed to be a primary component of matter at typical conditions where the neutron fugacity is of order 0.1-1, according to Saha's arguments, the mass fraction of $\alpha$ particles is dominated by a factor of $e^{Q / 13 k_{B} T}$, with the temperature $T$. In estimating such a fraction, however, no in-medium modification of nuclear masses except the

\footnotetext{
*e.nakano@kochi-u.ac.jp

†iida@kochi-u.ac.jp

‡whoriuchi@nucl.sci.hokudai.ac.jp
}

Coulomb corrections is normally considered. To deal with the in-medium modification, we will focus on a polaron picture, namely, a light cluster dressed by excitations in the medium.

The earliest theoretical investigations of hot nuclear matter are based on liquid-drop models [2]. The key ingredient of these models is mass formula for neutron-rich nuclei. Typically, in the presence of trapped electron neutrinos, nuclei in such matter (so-called supernova matter) are not extremely neutron-rich but too neutron-rich for their masses to be measured, which requires an extrapolation from empirical mass data. In the presence of internuclear Coulomb coupling, the Wigner-Seitz approximation for a lattice of nuclei embedded in a neutralizing background of electrons is often utilized. This approximation is known to give a good estimate of the lattice energy. Concerning the mass distribution, furthermore, a single species approximation in which only the nuclide that minimizes the system energy at fixed baryon density and lepton fraction occurs is often adopted for simplicity. This is good at sufficiently low temperatures. For better estimates of the mass distribution at temperatures relevant for supernova cores, the presence of $\alpha$ particles, etc., has been allowed for or even the nuclear statistical equilibrium has been imposed in some cases [3]. The most important upgrade has been made on how to calculate the nuclear mass itself as an extrapolation from the empirical data. The nucleon density profile in a Wigner-Seitz cell can be better predicted if one implements the Thomas-Fermi or Hartree-Fock theory [4-7]. Even in such predictions, the result still depends on uncertainties in the adopted equation of state of asymmetric nuclear matter, especially the density dependence of the symmetry energy. 
Experimentally, there can be two ways of studying properties of hot nuclear matter: heavy ion collisions at intermediate energy and quantum simulations with ultracold atoms. Data for such heavy ion collisions have already given some evidence for a liquid-gas phase transition of nuclear matter [8]. It is, however, important to note that the deduced temperature and density of finite matter (primary fragments) created in the collisions have some uncertainties, while finite-size corrections due to the Coulomb and surface effects have to be taken into consideration to deduce the critical point of bulk nuclear matter from the empirical data for the excitation energy, mass, and temperature of the primary fragments. Anyway, this is a direct way of probing nuclear matter. On the other hand, ultracold atoms provide us with an indirect way of probing nuclear matter. This is mainly because low density neutron matter, which is dominated by $s$-wave scattering with negative and large scattering length, is similar to trapped ultracold Fermi atoms near a Feshbach resonance $[9,10]$. It is thus expected that various superfluid properties of neutron matter such as the pairing gap, the BCS-BEC crossover, and vortices under rotation could be deduced from laboratory experiments, although the neutron-neutron effective range is fairly large as compared with the case of atoms. Incidentally, effects of a nonzero effective range of the interaction and nonzero proton fraction on the equilibrium properties of matter relevant to neutron stars have been studied by bearing in mind the quantum simulation in cold atomic systems [11,12]. Moreover, one can add impurity atoms to a system of ultracold Fermi atoms of a single species [13-15]. In the presence of interspecies interactions, these impurity atoms manifest themselves as polarons. The resultant atomic matter looks like cold nuclear matter of interest here. In relation to the present interest in the $\alpha$ particle as an impurity in cold neutron matter, there are cold atomic systems useful for the quantum simulation, e.g., a resonantly interacting atomic gas mixture of ${ }^{161}$ Dy and ${ }^{40} \mathrm{~K}$ [16], which has almost the same mass ratio with the $\alpha$-neutron system, a Fermi polaron interacting with medium via $p$-wave Feshbach resonance as well as background $s$-wave scattering [17,18], etc.

In the present study, under the motivation mentioned above, we evaluate in-medium modifications of an $\alpha$ particle that interacts with surrounding neutrons, by calculating its quasiparticle properties: the interaction energy, residue, effective mass, dispersion relation, and decay width. To this end, for simplicity, we employ an ideal situation where a single $\alpha$ particle is immersed in pure neutron matter that is in a normal state at zero temperature, rather than hot nuclear matter. Furthermore, we assume that the $\alpha$ particle is mobile with a small momentum and that the neutron matter is dilute enough, so that we can employ the low-energy treatment, i.e., the $\alpha$ particle is point like, while the interaction between the $\alpha$ particle and a surrounding neutron is described only by the $s$-wave scattering length. More quantitative discussion on these approximations will be given at the end of Sec. IV.
Note that such an $\alpha$ particle is not always stable. In fact, stability analysis of an $\alpha$ particle in neutron matter would require microscopic calculations, which will be addressed in the near future. We also remark that even before the experimental realization of trapped ultracold atoms, Kutschera and Wojcik [19] used to consider a proton impurity in neutron matter by sticking with the original polaron picture based on an electron-phonon system, which is different from the modern polaron picture based on a minority atom-majority atom system involving contact interactions.

We also note that $\alpha$-nucleon interactions in hot nuclear matter were considered in terms of excluded-volume effects [20] and the virial expansion [21]. Essentially, the $\alpha$-nucleon interactions considered in these approaches tend to be too repulsive and too attractive, respectively, when applied to a cold system of interest here. This is because in the former approach, no attraction is included, while in the latter approach, the second virial coefficient is related to the two-body phase shift dominated by the $p$-wave resonance.

It is interesting to note the possible relevance of the present polaron picture to $\alpha$ clustering, i.e., manifestation of $\alpha$ particle like configurations, in atomic heavy nuclei. Although there is no direct evidence for the presence of such $\alpha$ clustering, it is expected that the four-nucleon correlation responsible for the $\alpha$ clustering plays an essential role in describing the surface region of various heavy nuclei [22-24].

In such a dilute neutron-rich situation, two minority particles (protons) may tend to form an $\alpha$ particle by picking two neutrons out of the medium. Consequently, the system may look like pure neutron matter containing $\alpha$ particles as impurities. This situation may help us describe the neutron skin structure of heavy nuclei. The energy and decay rate of such $\alpha$ particles, if being known experimentally, could give us an opportunity of probing the bulk properties of the neutron medium.

This paper is organized as follows. In Sec. II we present the low-energy effective Hamiltonian for a single $\alpha$ particle embedded in neutron matter. In Sec. III we employ a variational method to obtain the energy of the $\alpha$ particle, which is equivalent to the self-energy calculation from the ladder type approximation. In Sec. IV we first employ empirical scattering data for setup of the parameters in the Hamiltonian and then discuss the numerical results for various quasiparticle properties as functions of neutron density, and also of the scattering length for general arguments. Section V is devoted to summary, physical consequences, and outlook.

\section{FORMULATION}

We consider a single $\alpha$ particle that is assumed to be an inert point particle and to be immersed in normal neutron matter at zero temperature. The Hamiltonian of this system is described by 


$$
\begin{aligned}
H(x)= & \sum_{s} \int \mathrm{d} r^{3} \psi_{s}^{\dagger}(r) \frac{-\nabla^{2}}{2 m} \psi_{s}(r)+\frac{1}{2} \sum_{s, t, s^{\prime}, t^{\prime}} \iint \mathrm{d} r^{3} \mathrm{~d} r^{\prime 3} \psi_{t^{\prime}}^{\dagger}\left(r^{\prime}\right) \psi_{s^{\prime}}^{\dagger}(r) V_{s^{\prime} t^{\prime} s t}\left(r-r^{\prime}\right) \psi_{s}(r) \psi_{t}\left(r^{\prime}\right)-\frac{\nabla_{x}^{2}}{2 M} \\
& +g \sum_{s} \int_{r} \psi_{s}^{\dagger}(r) \psi_{s}(r) \delta(r-x) \\
= & \sum_{s, p} \frac{p^{2}}{2 m} a_{s, p}^{\dagger} a_{s, p}+\frac{1}{2} \sum_{q, q^{\prime}, p, s, t, s^{\prime}, t^{\prime}} a_{t^{\prime}, q^{\prime}-p}^{\dagger} a_{s^{\prime}, q+p}^{\dagger} a_{s, q} a_{t, q^{\prime}} \tilde{V}_{s^{\prime} t^{\prime} s t}(p)-\frac{\nabla_{x}^{2}}{2 M}+g \sum_{p, q, s} a_{s, p}^{\dagger} a_{s, q} e^{-i(p-q) x}
\end{aligned}
$$

where $m(M)$ is the mass of a neutron (an $\alpha$ particle), and we have used the first quantization for the single $\alpha$ particle in the coordinate representation by $x$, and expanded the neutron field operator as $\psi_{s}(r)=\frac{1}{\sqrt{V}} \sum_{p} e^{i p r} a_{s, p}$ with the canonical relation $\left\{a_{s, p}^{\dagger}, a_{t, q}\right\}=\delta_{p, q} \delta_{s, t}$, where the subscript $s$ and $p$ represent the neutron spin and momentum, respectively. It is noted that the bare coupling constant $g$ for the $\alpha$-neutron interaction is related to the scattering length $a$ via the Lippmann-Schwinger equation in the low-energy limit:

$$
g^{-1}=\frac{m_{r}}{2 \pi \hbar^{2} a}-\sum_{p} \frac{2 m_{r}}{p^{2}}
$$

where $m_{r}{ }^{-1}=m^{-1}+M^{-1}$ is the reduced mass, and a momentum cutoff $\Lambda$ is assumed implicitly in the divergent integral $\sum_{p}$ as a regulator. The cutoff $\Lambda$ corresponds to the effective range $r_{0}$ via $r_{0} \approx \Lambda^{-1}$, and can be sent to infinity after the renormalization of the bare coupling constant $g$ in terms of the scattering length $a$ in Eq. (16). We use the natural units in which $\hbar=c=1$.

Now we implement a gauge transformation [25],

$$
S(x):=e^{i x \hat{P}} \text { with } \hat{P}=\sum_{s, p} p a_{s, p}^{\dagger} a_{s, p},
$$

which sends a gas of neutrons to the comoving frame of the impurity $\alpha$ particle. By using $S(x) a_{s, p} S^{-1}(x)=a_{s, p} e^{-i p x}$, the Hamiltonian (2) can be transformed to

$$
\begin{aligned}
S H(x) S^{-1}= & \sum_{s, p} \frac{p^{2}}{2 m} a_{s, p}^{\dagger} a_{s, p} \\
& +\frac{1}{2} \sum_{q, q^{\prime}, p, s, t} a_{t, q^{\prime}-p}^{\dagger} a_{s, q+p}^{\dagger} a_{s, q} a_{t, q^{\prime}} \tilde{V}_{s, t}(p) \\
& +\frac{\left(-i \nabla_{x}-\hat{P}\right)^{2}}{2 M}+g \sum_{p, q, s} a_{s, p}^{\dagger} a_{s, q},
\end{aligned}
$$

which satisfies the commutation relation $\left[S H(x) S^{-1},-i \nabla_{x}\right]=$ 0 , implying that after the transformation the momentum of the $\alpha$ particle represents the total momentum of the sys- tem. Therefore, we can replace the momentum operator by a $c$-number vector, i.e., $-i \nabla_{x} \rightarrow P$ in the transformed Hamiltonian,

$$
\begin{aligned}
S H(x) S^{-1} \rightarrow H_{\mathrm{eff}}(P)= & \sum_{s, p} \varepsilon_{p} a_{s, p}^{\dagger} a_{s, p}+\frac{(P-\hat{P})^{2}}{2 M} \\
& +g \sum_{p, q, s} a_{s, p}^{\dagger} a_{s, q},
\end{aligned}
$$

where we have furthermore dropped the $\tilde{V}_{s, t}$ by assuming that the neutron self-interaction effects are incorporated in the single-particle energy $\varepsilon_{p}=\frac{p^{2}}{2 m^{*}}+U$. Here, $m^{*}$ is the effective mass in medium, but we assume that the in-medium modification is negligible and take the same notation, i.e., $m^{*}=m$ hereafter. $U$ is the density dependent interaction energy per particle, which, e.g., can be deduced from a Thomas-Fermi approach [26] but is not relevant for the present study. We will use the above Hamiltonian (6) in the following calculations.

\section{SINGLE PARTICLE-HOLE PAIR APPROXIMATION}

To describe excitations accompanied by the impurity $\alpha$ particle, we implement a variational method in which the variational state is spanned by a single particle-hole $(\mathrm{p}-\mathrm{h})$ pair excitation near the neutron Fermi surface [27,28]:

$$
|\Psi\rangle=F_{0}\left|\psi_{0}\right\rangle+\sum_{k>, p<, s} F_{k, p}^{s} a_{s, k}^{\dagger} a_{s, p}\left|\psi_{0}\right\rangle,
$$

where $\left|\psi_{0}\right\rangle$ is the state occupied by neutrons up to the Fermi momentum $k_{F}, k>(p<)$ denotes $|k|>k_{F}\left(|p|<k_{F}\right)$, and $F_{0}$ and $F_{k, p}^{s}$ are variational parameters. In fact, $F_{k, p}^{s}$ serves as the wave function of the $\mathrm{p}$-h pair of the corresponding momentum and spin. We remark in passing that even such a lowest-order form of the variational state can well reproduce the empirical energy and effective mass of an impurity that repulsively interacts with medium fermions in the vicinity of the unitarity limit, i.e., $|a| \rightarrow \infty$ and zero effective range [15].

The expectation value of the transformed Hamiltonian (6) with respect to the state (7) gives

$$
\left\langle H_{\mathrm{eff}}(P)\right\rangle=\sum_{s, q} \varepsilon_{q}\left\langle a_{s, q}^{\dagger} a_{s, q}\right\rangle+\frac{\left\langle(P-\hat{P})^{2}\right\rangle}{2 M}+g \sum_{q, q^{\prime}, s}\left\langle a_{s, q}^{\dagger} a_{s, q^{\prime}}\right\rangle,
$$

where $\langle\cdots\rangle=\langle\Psi|\cdots| \Psi\rangle$, and each term in the right side is given, respectively, by

$$
\sum_{q} \varepsilon_{q}\left\langle a_{s, q}^{\dagger} a_{s, q}\right\rangle=\sum_{q<} \varepsilon_{q}\left(\left|F_{0}\right|^{2}+\sum_{k>, p<t}\left|F_{k, p}^{t}\right|^{2}\right)+\sum_{k>, p<}\left(\varepsilon_{k}-\varepsilon_{p}\right)\left|F_{k, p}^{s}\right|^{2},
$$




$$
\begin{aligned}
\left\langle(P-\hat{P})^{2}\right\rangle & =P^{2}\left|F_{0}\right|^{2}+\sum_{k>, p<, s}\left|F_{k, p}^{s}\right|^{2}\left[P^{2}-2 P \cdot(k-p)+(k-p)^{2}\right] \\
\text { and } \sum_{q, q^{\prime}}\left\langle a_{s, q}^{\dagger} a_{s, q^{\prime}}\right\rangle & =\sum_{q<}\left(\left|F_{0}\right|^{2}+\sum_{k>, p<, t}\left|F_{k, p}^{t}\right|^{2}\right)+\sum_{k>, p<}\left(F_{0} F_{k, p}^{s *}+F_{0}^{*} F_{k, p}^{s}\right)+\sum_{q>, q^{\prime}>, p<} F_{q, p}^{s *} F_{q^{\prime}, p}^{s}-\sum_{k>, q<, q^{\prime}<} F_{k, q}^{s *} F_{k, q^{\prime}}^{s} .
\end{aligned}
$$

\section{A. Quasiparticle energy}

We impose the normalization condition by using a Lagrange multiplier $\mu$ that turns out to be the ground state energy $E_{P}$ of the system with momentum $P$. In fact, $E_{P}$ represents the $\alpha$ particle dispersion relation up to the Fermi energy of the medium neutrons. The variational condition $\delta\left\langle H_{\text {eff }}-\mu\right\rangle=0$ leads to a set of eigenvalue equations, ${ }^{1}$

$$
\begin{aligned}
\frac{P^{2}}{2 M} F_{0}+\sum_{q<, s} g\left(F_{0}+\sum_{k>} F_{k, q}^{s}\right) & =\omega F_{0}, \\
\Omega_{k, p ; P}^{s} F_{k, p}^{s}+g\left(F_{0}+\sum_{q^{\prime}>} F_{q^{\prime}, p}^{s}-\sum_{q^{\prime}<} F_{k, q^{\prime}}^{s}\right) & =\omega F_{k, p}^{s},
\end{aligned}
$$

where $\omega=\mu-\sum_{q<, s} \varepsilon_{q}$, and

$$
\Omega_{k, p ; P}^{s}:=\varepsilon_{k}-\varepsilon_{p}+\frac{P^{2}-2 P \cdot(k-p)+(k-p)^{2}}{2 M}+g \sum_{q<} .
$$

From the Lippmann-Schwinger equation (3), the bare coupling constant $g$ turns out to be vanishingly small as a negative power of the momentum cutoff $\Lambda$. In the renormalization procedure in terms of the scattering length [27], therefore, we will drop the terms $g \sum_{q^{\prime}<} F_{k, q^{\prime}}^{s}$ in Eq. (13) and $g \sum_{q<}$ in $\Omega_{k, p ; P}^{s}$ as subleading order.

Using the auxiliary field $\chi_{p}^{s}=F_{0}+\sum_{q^{\prime}>} F_{q^{\prime}, p}^{s}$ in Eqs. (12)-(13), we obtain the following equation:

$$
\omega=\frac{P^{2}}{2 M}+\Sigma(\omega, P)
$$

with $\Sigma(\omega, P)=\sum_{p<, s} \frac{1}{\frac{m_{r}}{2 \pi a}-\sum_{k>}\left(\frac{1}{\omega-\Omega_{k, p ; P}^{s}}+\frac{2 m_{r}}{k^{2}}\right)-\sum_{q<\frac{2 m_{r}}{q^{2}}}}$,

from which the eigenvalues of $\omega$ can be determined. In this equation, $\Sigma(\omega, P)$ can be interpreted as the self-energy obtained from the non-self-consistent ladder approximation for the $\alpha$-neutron scattering amplitude [28]. In the case of a repulsive interaction $a>0$ of interest here, the resulting positive energy state is characterized by the outgoing scattering amplitude. The real part of the corresponding quasiparticle energy

\footnotetext{
${ }^{1}$ One can also obtain the same result from a time-dependent variational approach to the Dirac type effective action, i.e., $\delta\langle\Psi(t)| i \partial_{t}-$ $H_{\text {eff }}|\Psi(t)\rangle=0$, if one assumes $|\Psi(t)\rangle \approx e^{-i \mu t}$. Note that this $|\Psi(t)\rangle$ includes excited states, as well as the ground state.
}

$E_{P}$ can thus be obtained from the spectral peak as

$$
E_{P}=\frac{P^{2}}{2 M}+\operatorname{Re} \Sigma\left(E_{P}+i 0, P\right),
$$

where the analytic continuation to the upper half-plane $\omega \rightarrow$ $\omega+i 0$ has been made; it traces back to the (retarded) propagator of the $\alpha$ particle that undergoes multiple scattering.

\section{B. Quasiparticle residue, width, and effective mass}

Validity of the quasiparticle picture for the $\alpha$ particle requires a finite residue and a relatively small width (long lifetime) compared with the real part of quasiparticle energy, both of which imply that the propagator of the $\alpha$ particle near its pole behaves as

$$
G^{R}(\omega, P)=\frac{1}{\omega+i 0-\frac{P^{2}}{2 M}-\Sigma(\omega+i 0, P)} \approx \frac{Z_{P}}{\omega-E_{P}+i \Gamma_{P}},
$$

where the width is given approximately by the imaginary part, $\Gamma_{P}=-Z_{P} \operatorname{Im} \Sigma\left(E_{P}+i 0, P\right)$, and the quasiparticle residue is defined by ${ }^{2}$

$$
Z_{P}=\left[1-\left.\operatorname{Re} \frac{\partial \Sigma(\omega+i 0, P)}{\partial \omega}\right|_{\omega=E_{P}}\right]^{-1} .
$$

The effective mass $M^{*}$, which characterizes the mobility of the $\alpha$ particle in the medium, is defined in the momentum expansion around the pole as

$$
\begin{aligned}
M^{*} & :=\left(\left.\frac{\mathrm{d}^{2} E_{P}}{\mathrm{~d} P^{2}}\right|_{P=0}\right)^{-1} \\
& =\frac{M}{Z}\left[1+\left.M \frac{\partial^{2} \operatorname{Re} \Sigma(\omega+i 0, P)}{\partial P^{2}}\right|_{\omega=E, P=0}\right]^{-1},
\end{aligned}
$$

where $E=E_{P=0}$ and $Z=Z_{P=0}$. As will be shown by numerical results below, the quasiparticle picture is indeed valid for the parameter region that corresponds to the low density and large isospin asymmetric situation considered in this study.

\footnotetext{
${ }^{2}$ Unlike unitary cold atoms there is no bound state between an $\alpha$ particle and a neutron. In fact, a resonant ${ }^{5} \mathrm{He}(3 / 2-)$, if appearing in vacuum, would be very unstable to $p$-wave dissociation into an $\alpha$ particle and a neutron, while in the $s$-wave channel there is no positive scattering length state that can be obtained continuously from the negative scattering length state (attractive branch). In the absence of decay to the attractive branch, therefore, the present positive energy state is not an excited one.
} 
The quasiparticle dispersion of the $\alpha$ particle can thus be well approximated by

$$
E_{P} \simeq E+\frac{P^{2}}{2 M^{*}}
$$

near the low energy limit.

\section{NUMERICAL RESULTS AND DISCUSSION}

Before exhibiting numerical results for the quantities described in the previous section, we give typical reference values of the scattering length and the neutron density as

$$
\begin{aligned}
a_{\text {ref }} & =2.64 \mathrm{fm}, \\
\rho_{\text {ref }} & =0.01 \rho_{0}, \quad \rightarrow \quad k_{F \text { ref }}=0.36 \mathrm{fm}^{-1},
\end{aligned}
$$

where $\rho_{0}=0.16 \mathrm{fm}^{-3}$ is the normal nuclear density. These values lead to the dimensionless coupling parameter $a_{\text {ref }} k_{F \text { ref }}=0.95 \simeq 1$, which marginally correspond to the strong coupling regime. The value of $\rho_{\text {ref }}$ is supposed to be of order the typical density above which neutrinos are trapped in supernova cores [1], but lower than the typical density in the neutron skin of heavy nuclei [29]. The value of the scattering length has been determined from the $\alpha$ neutron potential [30] that reproduces the experimental phase shift for low-energy $\alpha$-neutron scattering cross section. In the course of this determination, the effective range has been simultaneously obtained as $r_{0}=1.43 \mathrm{fm}$. This value gives $r_{0} k_{F \text { ref }}=0.51<1$, which is not so small but still implies the validity of the present low-energy treatment of the $\alpha$-neutron interaction in terms of the zero-range potential characterized by the scattering length (3) alone. We remark that the values of $a_{\text {ref }}$ and $r_{0}$ obtained here are consistent with the earlier results [31,32]. Finally, we set the bare mass ratio of the $\alpha$ particle as

$$
\frac{M}{m}=4 \text {. }
$$

For these reference values of the parameters given above, the numerical results for quasiparticle properties of the $\alpha$ particle at $P=0$ are given as follows:

$$
\begin{aligned}
E_{\mathrm{ref}} & =0.467 \varepsilon_{F \mathrm{ref}}=1.26 \mathrm{MeV}, \\
Z_{\mathrm{ref}} & =0.650, \\
M_{\mathrm{ref}}^{*} & =1.217 M, \\
\text { and } \Gamma_{\mathrm{ref}} & =0.032 \varepsilon_{F \text { ref }}=0.086 \mathrm{MeV},
\end{aligned}
$$

where $\varepsilon_{F \text { ref }}=\frac{(\hbar c)^{2} k_{\text {ref }}^{2}}{2 m c^{2}}=2.69 \mathrm{MeV}$. The interaction energy of the $\alpha$ particle at rest, $E_{\text {ref }}$, is positive, a feature that reflects the positive scattering length corresponding to repulsion, and is much larger than the decay width. As can be interpreted from the diagrammatic representation of the present self-energy, the decay width comes solely from the process in which the $\alpha$ particle, a quasiparticle dressed by a cloud of p-h bubbles, decays into a bare $\alpha$ particle and a neutron in the $s$-wave scattering state. In addition, the quasiparticle residue obtained here is fairly close to unity. All these results support the quasiparticle picture presumed in this study. At the reference neutron density the effective mass increases by about $20 \%$ from its vacuum value. Such increase seems like a general

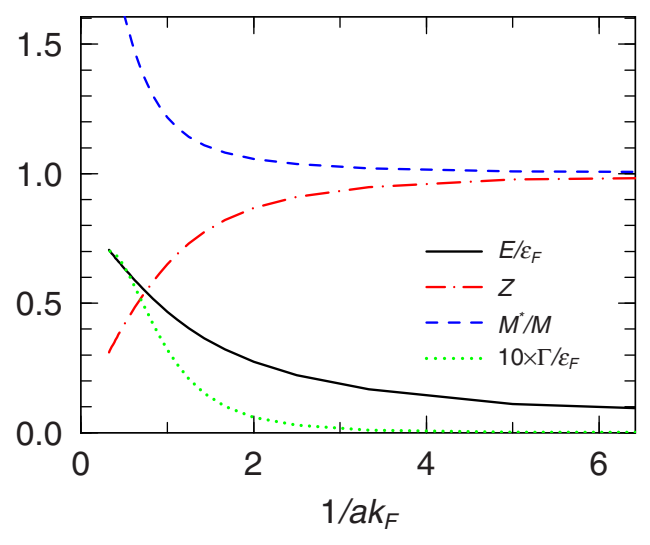

FIG. 1. Energy, residue, and mass ratio of an $\alpha$ particle calculated as functions of $1 / a k_{F}$ at $P=0$.

feature of various impurity-medium combinations, irrespective of whether the impurity-medium interaction is repulsive or attractive. While the scattering length (22) employed here is an empirical one, for more general discussion, we extend our calculations and present in Fig. 1 the results as functions of the dimensionless coupling.

For weak coupling the quasiparticle picture works obviously. It is also found from the figure that while even in the strong coupling regime $1 / a k_{F} \ll 1$, the relation $E \gg \Gamma$ still holds, in such a regime the quasiparticle is less identifiable due to the smallness of the residue.

Now we show in Fig. 2 the full dispersion relation $E_{P}$ obtained from Eq. (17) with the reference values of the parameters, together with the approximate one (21) expressed by the effective mass.

We observe that the appreciable $P$ dependence of the self-energy $\operatorname{Re} \Sigma$, also plotted in the figure, accounts for the deviation between the full and the approximate dispersion relations for finite $P$. The deviation is nevertheless small enough that the approximate one seems to work for a relatively wide range of the momentum.

Finally we show how the quasiparticle properties depend on the neutron density.

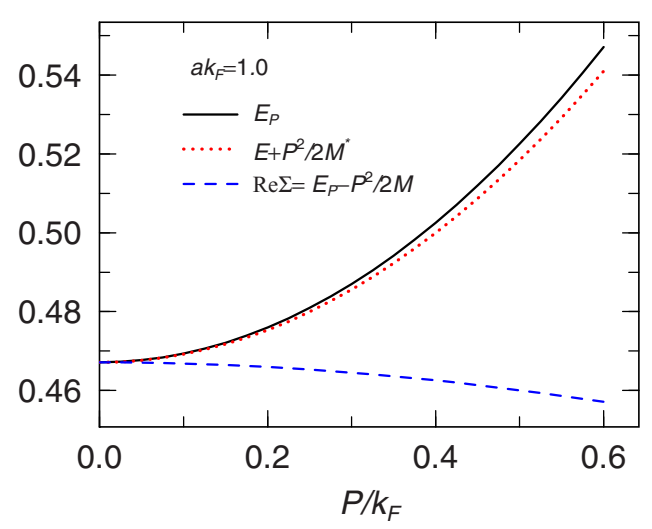

FIG. 2. Dispersion relations of an $\alpha$ particle calculated in units of $\varepsilon_{F}$ at $a k_{F}=1.0 \simeq a_{\text {ref }} k_{F \text { ref }}$. 


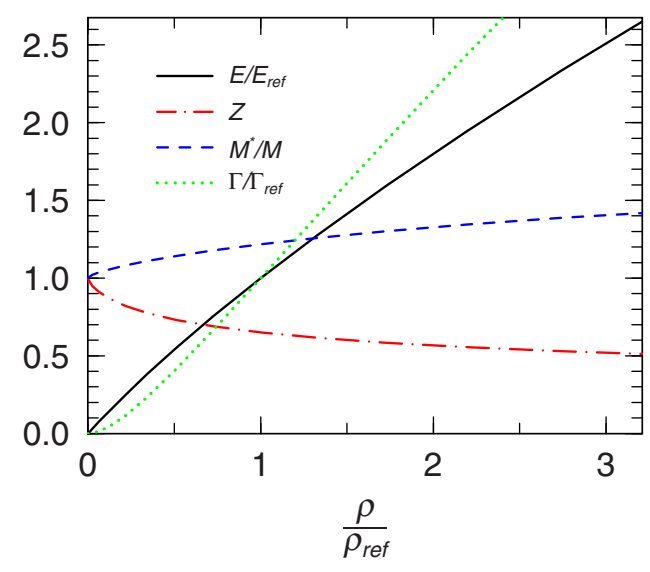

FIG. 3. Neutron density dependence of the energy, residue, effective mass, and width of an $\alpha$ particle calculated at $a k_{F \text { ref }}=1.0$.

Since the increase in the density extends the phase space available for the $\mathrm{p}$-h fluctuations, the interaction energy and the decay width increase with density, as can be seen in Fig. 3. On the other hand, the relation $E \gg \Gamma$ is kept, and eventually at $\rho=4.2 \rho_{\text {ref }}=0.0067 \mathrm{fm}^{-3}$, the kinetic energy of the background neutron gas reaches $\varepsilon_{F}=\varepsilon_{F \text { ref }}\left(\frac{\rho}{\rho_{\text {ref }}}\right)^{2 / 3}=7$ $\mathrm{MeV}$, which is added to the kinetic energy of the constituent neutrons inside the $\alpha$ particle, and thus cancels the binding energy of the $\alpha$ particle per nucleon $\approx 7 \mathrm{MeV}$. At this stage, the stability of the $\alpha$ particle itself becomes doubtful. More realistically, the neutron separation energy of ${ }^{4} \mathrm{He}$ in vacuum, which amounts to $20.6 \mathrm{MeV}$, helps us to give a better estimate of the critical neutron density at which the $\alpha$ particle is no longer bound. At the neutron density of about $21 \rho_{\text {ref }} \simeq 0.034 \mathrm{fm}^{-3}$, the neutron Fermi energy reaches $20.6 \mathrm{MeV}$. Interestingly, a microscopic calculation for the energy of the four-nucleon system in symmetric nuclear matter [33] has shown by comparison with the free four-nucleon energy threshold in the medium that the $\alpha$-like cluster dissociates at $\rho=0.03 \mathrm{fm}^{-3}$ due to Pauli blocking effects at zero temperature, which is close to the above neutron density.

In what follows we discuss the negligibility of higher partial-wave contributions than the $s$ wave. In general the scattering $T$ matrix and its partial-wave decomposition [34] can be obtained from the Lippmann-Schwinger equation as

$$
T\left(\mathbf{k}, \mathbf{k}^{\prime}\right)=\sum_{l=0,1,2, \cdots}(2 l+1) T_{l}(k) P_{l}\left(\hat{k} \hat{k}^{\prime}\right)
$$

with $P_{l}(x)$ being the Legendre polynomials, and the partialwave matrix can be expanded, at low scattering energy $E_{\text {rel }}=$ $k^{2} / 2 m_{r}$ and for finite range two-body potentials, as

$$
T_{l}(k)=\frac{2 \pi}{m_{r}} \frac{k^{2 l}}{a_{l}^{-1}-r_{l} k^{2} / 2+\mathcal{O}\left(k^{4}\right)+i k^{2 l+1}},
$$

where $a_{l}$ and $r_{l}$ correspond respectively to the generalized scattering length and effective range just like the case of the $s$ wave; $a_{0}$ is identical with $a$. The quasiparticle poles for possible bound/resonance states at low energies can be obtained in terms of these parameters. Now let us give a ballpark estimate of the density region in which the $s$ wave dominates in the scattering process: Comparing the $s$ - and $p$-wave matrices with the empirical scattering length (volume) at the neutron Fermi momentum, the condition $\left|T_{0}\right|>\left|T_{1}\right|$ reduces to

$$
a_{0}>\left|a_{1}\right|\left(\frac{m_{r}}{m}\right)^{2} k_{F}^{2} \rightarrow k_{F}<0.25 \mathrm{fm}^{-1} \rightarrow \rho<0.003 \rho_{0},
$$

which is compatible with the validity condition for the Taylor expansion in Eq. (30), i.e., $2\left|a_{0}\right|^{-1}>\left|r_{0}\right|\left(m_{r} / m\right)^{2} k_{F}^{2}$ and $2\left|a_{1}\right|^{-1}>\left|r_{1}\right|\left(m_{r} / m\right)^{2} k_{F}^{2}$. Here, we have used $a_{0}=$ $2.64 \mathrm{fm}\left(a_{1}=a_{P_{3 / 2}}=-67.1 \mathrm{fm}^{3}\right)$ and $r_{0}=1.43 \mathrm{fm}\left(r_{1}=\right.$ $r_{P_{3 / 2}}=-0.87 \mathrm{fm}^{-1}$ ) for the $s$-( $p$-)wave scattering amplitude, obtained from the phenomenological $\alpha-n$ potential and scattering data in vacuum [30]. Furthermore, the $p$-wave $\left(P_{3 / 2}\right)$ resonance in the $\alpha-n$ system appears at the center-of-mass neutron kinetic energy $\approx 0.9 \mathrm{MeV}[21,31]$, which amounts to the neutron Fermi momentum $k_{F} \approx 0.26 \mathrm{fm}^{-1}$ when the $\alpha$ particle is at rest. At densities higher than the one corresponding to $k_{F} \approx 0.26 \mathrm{fm}^{-1}$, the $P_{3 / 2}$ resonance $\left({ }^{5} \mathrm{He}\right)$ may survive strong decay long enough to constitute a fraction of the matter under the influence of Pauli blocking effect [35], although this is a scenario valid if and only if the $\alpha$ particle itself is bound in neutron matter and also the resonance energy is the same as in vacuum. We remark in passing that if a similar argument can be applied to the temperature direction, the present model is valid at temperatures below both about $1 \mathrm{MeV}$ and the neutron Fermi temperature.

The above estimates indicate that the present model, which neglects higher waves, is safely applicable only at very low neutron densities $\rho<0.003 \rho_{0}$, although this bound is obtained from the extrapolation from the zero density limit using the two-body scattering data in vacuum. Nevertheless, we stress that the quasiparticle picture of the $\alpha$ particle, which is obtained from the $s$-wave pole structure in this study, seems hardly disturbed by the other higher-wave contributions, as seen from the independence of different $T_{l}$ 's in Eq. (29) that is valid even at finite densities. For realistic description of the system at even higher densities, of course, we have to pursue the quasiparticle poles from the $p$ - and higher-wave matrices $T_{l>0}$ as well as the $s$-wave one, and compare quasiparticle energies and strengths from these poles to figure out which one dominates at given density, or we can calculate the $T$ matrix directly using an empirical two-body potential without the partial-wave decomposition. This is, however, out of the present scope.

We finally remark that in a very wide density regime below the normal nuclear density, zero-temperature supernova matter at proton fraction of order 0.3 can satisfy the condition that the Fermi momentum of a neutron gas outside nuclei is below $0.25 \mathrm{fm}^{-1}$ (see Fig. 4 in Ref. [36]). We thus believe that the present model is fairly reasonable from a phenomenological point of view.

\section{SUMMARY AND OUTLOOK}

In this study we elucidate the quasiparticle properties of a single $\alpha$ particle immersed in a cold dilute neutron gas by evaluating the self-energy from the variational treatment equivalent to a non-self-consistent ladder approximation that 
incorporates an empirical value of the $\alpha$-neutron low-energy $s$-wave scattering length (22). Our result shows that adding a single $\alpha$ particle into a dilute neutron gas costs at least the interaction energy (25) that we calculated for the $\alpha$ particle at rest. Note that such interaction energy reads $E=0.467 \varepsilon_{F}=$ $\frac{2.23}{m} \rho^{2 / 3}$. This could be useful to deduce the fraction of $\alpha$ particles in supernova matter. It is also interesting to note that the effective mass calculated with the same parameter set leads to an approximate dispersion relation of the $\alpha$ particle, $E_{p}=E+P^{2} / 2 M^{*}$, with the density-dependent interaction energy $E$ given above. Applying the dispersion relation to the Bose distribution for a dilute $\alpha$ gas in neutron matter, we can estimate the transition temperature for possible Bose-Einstein condensation [37] to be $T_{\mathrm{BEC}}=\frac{2 \pi \hbar^{2}}{M^{*}}\left(\frac{\rho_{\alpha}}{\zeta(3)}\right)^{2 / 3} \mathrm{MeV}$, which gives $T_{\mathrm{BEC}}=0.14 \mathrm{MeV}$ at $\rho_{\alpha}=\rho_{\text {ref }} / 10$. This might have some relevance to $\alpha$ clustering in the surface of heavy nuclei.

In the present work the $\alpha$ particle in neutron matter is treated as an inert point particle, i.e., the inner structure is neglected from the low-energy point of view. To go beyond it, the $\alpha$ particle has to be regarded as a cluster of four nucleons. Then, diagrammatically needed is to take into account neutron exchange in and out of the cluster in scattering processes as well as deuteron-like pair correlations, which might eventually break up the $\alpha$ particle at sufficiently high densities [38-40]. For elaborate studies to clarify the stability of such $\alpha$ clustering in neutron matter, the wave function of the system is needed in terms of interacting nucleons with phenomenological potentials [26,33].

As discussed in the previous section, the present study is restricted to the dilute limit of the $\alpha$ density and to the cold, isospin asymmetric limit of the nuclear medium. Next it is interesting to consider the system of two $\alpha$ particles in neutron matter. The interaction between the $\alpha$ particles, which resembles that between two repulsive Fermi polarons in the context of ultracold atoms except for the Coulomb repulsion, has some possible relevance to neutrino scattering off light clusters in supernova matter. To make better estimates, it would be necessary to raise both the proton fraction and temperature [41] in the nuclear medium and also consider screening corrections to the $\alpha-\alpha$ Coulomb repulsion [42].

\section{ACKNOWLEDGMENTS}

We are grateful to T. Hatsuda, P. Naidon, H. Tajima, T. Uesaka, J. Zenihiro, K. Nakazato, and H. Togashi for useful discussion. This work was in part supported by Grants-in-Aid for Scientific Research through Grants No. 17K05445, No. 18K03635, No. 18H01211, No. 18H04569, No. 18H05406, and No. 19H05140, provided by JSPS. E.N. acknowledges the hospitality of the Institut für Theoretische Physik, Goethe Universität Frankfurt, where this work was completed. W.H. acknowledges the Collaborative Research Program 2020, Information Initiative Center, Hokkaido University.

\section{APPENDIX A: DIMENSIONLESS EXPRESSION FOR THE SELF-ENERGY}

It is convenient to rewrite the self-energy in terms of dimensionless quantities (symbols with tilde) as well as $k_{F}$ and $\varepsilon_{F}$ as

$$
\begin{aligned}
\Sigma(\omega+i 0, P) & =\sum_{p<} \frac{1}{g_{r}^{-1}-G(\omega+i 0, P ; p)-\sum_{q<} \frac{2 m_{r}}{q^{2}}} \\
& =(2 \pi)^{2} \varepsilon_{F} \sum_{\rho<} \frac{\tilde{g}^{-1}-\operatorname{Re} \tilde{G}+i \operatorname{Im} \tilde{G}}{\left(\tilde{g}^{-1}-\operatorname{Re} \tilde{G}\right)^{2}+\operatorname{Im}^{2} \tilde{G}}
\end{aligned}
$$

with

$$
G(\omega, P ; p) \equiv \sum_{k>}\left\{\left[\omega-\varepsilon_{k}+\varepsilon_{p}-\frac{P^{2}-2 P(k-p)+(k-p)^{2}}{2 M}\right]^{-1}+\left[\frac{k^{2}}{2 m_{r}}\right]^{-1}\right\}
$$

Here, we have factored out the dimensional coefficients as $G=\frac{k_{F}^{3}}{(2 \pi)^{2} \varepsilon_{F}} \tilde{G}$ and $g_{r}^{-1}=\frac{k_{F}^{3}}{(2 \pi)^{2} \varepsilon_{F}} \tilde{g}^{-1}$, and expressed the rest parts as

$$
\begin{aligned}
\tilde{G}(\omega, P ; p-P) & =\int_{1}^{\infty} \mathrm{d} \kappa \kappa^{2} \int_{-1}^{1} \mathrm{~d} x\left\{\left[\mathcal{E}-\kappa^{2}+\bar{\rho}^{2}-\frac{\kappa^{2}+\rho^{2}-2 \kappa \rho x}{R}+i 0\right]^{-1}+\left[\kappa^{2}\left(R^{-1}+1\right)\right]^{-1}\right\} \\
\text { and } \quad \tilde{g}^{-1} & =2 \pi^{2} \frac{R}{R+1}\left(\frac{1}{2 \pi a k_{F}}-\sum_{\rho<} \frac{2}{\rho^{2}}\right)
\end{aligned}
$$

where $\bar{\rho} \equiv \sqrt{(p-P)^{2}} / k_{F}$, and we have introduced variables: $\mathcal{E}=\omega / \varepsilon_{F}, \mathcal{P}=P / k_{F}, R=M / m,|p| / k_{F}=\rho$, and $|k| / k_{F}=\kappa$, and shifted the momentum temporarily, $p \rightarrow p-P$, for convenience. Performing the $x$ integration in Eq. (A3) first, we obtain

$$
\begin{aligned}
\int_{-1}^{1} \mathrm{~d} x\left[\mathcal{E}-\frac{R \kappa^{2}-R \bar{\rho}^{2}+\kappa^{2}+\rho^{2}-2 \kappa \rho x}{R}+i 0\right]^{-1}= & \frac{R}{2 \kappa \rho}\left[\ln \left|\frac{\left(\kappa-\kappa_{-}\right)\left(\kappa-\kappa_{+}\right)}{\left(\kappa+\kappa_{-}\right)\left(\kappa+\kappa_{+}\right)}\right|\right. \\
& \left.-i \pi \theta\left(1-\left|\frac{R \mathcal{E}-R \kappa^{2}+R \bar{\rho}^{2}-\kappa^{2}-\rho^{2}}{2 \kappa \rho}\right|\right)\right],
\end{aligned}
$$

where $\theta(x)$ is the Heaviside function, and

$$
\kappa_{ \pm} \equiv \frac{\rho}{R+1} \pm \frac{1}{R+1} \sqrt{R(R+1) \bar{\rho}^{2}-R \rho^{2}+\mathcal{E} R(R+1)} .
$$


Note that $\kappa_{+} \geqslant 0$ and $\kappa_{-} \leqslant 0$ for $R>1$ and $\mathcal{E}>0$. Then, the real and imaginary parts read, respectively as

$$
\begin{aligned}
\operatorname{Re} \tilde{G}(\omega, P ; p-P) & =\int_{1}^{\infty} \mathrm{d} \kappa\left[\frac{R \kappa}{2 \rho} \ln \left|\frac{\left(\kappa-\kappa_{-}\right)\left(\kappa-\kappa_{+}\right)}{\left(\kappa+\kappa_{-}\right)\left(\kappa+\kappa_{+}\right)}\right|+2 \frac{R}{R+1}\right] \\
& =\frac{R}{4 \rho}\left[\left(1-\kappa_{+}^{2}\right) \ln \left|\frac{1+\kappa_{+}}{1-\kappa_{+}}\right|+\left(1-\kappa_{-}^{2}\right) \ln \left|\frac{1+\kappa_{-}}{1-\kappa_{-}}\right|\right]-\frac{R}{R+1}
\end{aligned}
$$

and

$$
\begin{aligned}
\operatorname{Im} \tilde{G}(\omega, P ; p-P) & =-\pi \int_{1}^{\infty} \mathrm{d} \kappa \frac{R \kappa}{2 \rho} \theta\left(1-\left|\frac{R \mathcal{E}-R \kappa^{2}+R \bar{\rho}^{2}-\kappa^{2}-\rho^{2}}{2 \kappa \rho}\right|\right) \\
& =-\frac{R \pi}{4 \rho} \theta\left(\kappa_{+}-1\right)\left[\kappa_{+}^{2}-\kappa_{-}^{2} \theta\left(-\kappa_{-}-1\right)-\theta\left(1+\kappa_{-}\right)\right] .
\end{aligned}
$$

The $p$ integration in Eq. (A1) can be done numerically after shifting back $p-P \rightarrow p$.

[1] H. A. Bethe, Rev. Mod. Phys. 62, 801 (1990).

[2] D. Q. Lamb, J. M. Lattimer, C. J. Pethick, and D. G. Ravenhall, Phys. Rev. Lett. 41, 1623 (1978).

[3] N. Buyukcizmeci et al., Nucl. Phys. A 907, 13 (2013).

[4] P. Bonche and D. Vautherin, Nucl. Phys. A 372, 496 (1981).

[5] M. Barranco and J.-R. Buchler, Phys. Rev. C 24, 1191 (1981).

[6] R. Ogasawara and K. Sato, Prog. Theor. Phys. 70, 1569 (1983).

[7] H. Togashi et al., Nucl. Phys. A 961, 78 (2017).

[8] J. Pochodzalla et al., Phys. Rev. Lett. 75, 1040 (1995).

[9] N. Navon et al., Science 328, 729 (2010).

[10] M. Horikoshi and M. Kuwata-Gonokami, Int. J. Mod. Phys. E 28, 1930001 (2019).

[11] P. van Wyk, H. Tajima, D. Inotani, A. Ohnishi, and Y. Ohashi, Phys. Rev. A 97, 013601 (2018).

[12] H. Tajima, T. Hatsuda, P. van Wyk, and Y. Ohashi, Sci. Rep. 9, 18477 (2019).

[13] A. Schirotzek, C.-H. Wu, A. Sommer, and M. W. Zwierlein, Phys. Rev. Lett. 102, 230402 (2009).

[14] C. Kohstall, M. Zaccanti, M. Jag, A. Trenkwalder, P. Massignan, G. M. Bruun, F. Schreck, and R. Grimm, Nature 485, 615 (2012).

[15] F. Scazza, G. Valtolina, P. Massignan, A. Recati, A. Amico, A. Burchianti, C. Fort, M. Inguscio, M. Zaccanti, and G. Roati, Phys. Rev. Lett. 118, 083602 (2017).

[16] C. Ravensbergen, E. Soave, V. Corre, M. Kreyer, B. Huang, E. Kirilov, and R. Grimm, Phys. Rev. Lett. 124, 203402 (2020).

[17] E. Wille, F. M. Spiegelhalder, G. Kerner, D. Naik, A. Trenkwalder, G. Hendl, F. Schreck, R. Grimm, T. G. Tiecke, J. T. M. Walraven, S. J. J. M. F. Kokkelmans, E. Tiesinga, and P. S. Julienne, Phys. Rev. Lett. 100, 053201 (2008).

[18] J. Levinsen, P. Massignan, F. Chevy, and C. Lobo, Phys. Rev. Lett. 109, 075302 (2012).

[19] M. Kutschera and W. Wójcik, Phys. Rev. C 47, 1077 (1993).

[20] S. Lalit, M. A. A. Mamun, C. Constantinou, and M. Prakash, Eur. Phys. J. A 55, 10 (2019).

[21] C. J. Horowitz and A. Schwenk, Nucl. Phys. A 776, 55 (2006).
[22] D. M. Brink, in Many-Body Description of Nuclear Structure and Reactions, Proceedings of the International School of Physics "Enrico Fermi," Course 36, edited by Claude Bloch (Academic Press, New York, 1966).

[23] D. M. Brink and J. J. Castro, Nucl. Phys. A 216, 109 (1973).

[24] S. Yang, C. Xu, G. Röpke, P. Schuck, Z. Ren, Y. Funaki, H. Horiuchi, A. Tohsaki, T. Yamada, and B. Zhou, Phys. Rev. C 101, 024316 (2020).

[25] T. D. Lee, F. E. Low, and D. Pines, Phys. Rev. 90, 297 (1953).

[26] K. Oyamatsu and K. Iida, Prog. Theor. Phys. 109, 631 (2003).

[27] F. Chevy, Phys. Rev. A 74, 063628 (2006).

[28] R. Combescot, A. Recati, C. Lobo, and F. Chevy, Phys. Rev. Lett. 98, 180402 (2007).

[29] S. Typel, Phys. Rev. C 89, 064321 (2014).

[30] H. Kanada, T. Kaneko, S. Nagata, and M. Nomoto, Prog. Theor. Phys. 61, 1327 (1979).

[31] R. A. Arndt, D. L. Long, and L. D. Roper, Nucl. Phys. A 209, 429 (1973).

[32] R. Kamouni and D. Baye, Nucl. Phys. A 791, 68 (2007).

[33] G. Röpke, P. Schuck, Y. Funaki, H. Horiuchi, Z. Ren, A. Tohsaki, C. Xu, T. Yamada, and B. Zhou, Phys. Rev. C 90, 034304 (2014).

[34] J. R. Taylor, Scattering Theory (John Wiley, New York, 1972).

[35] G. Röpke, Phys. Rev. C 101, 064310 (2020).

[36] B. Schuetrumpf, K. Iida, J. A. Maruhn, and P.-G. Reinhard, Phys. Rev. C 90, 055802 (2014).

[37] X.-H. Wu, S.-B. Wang, A. Sedrakian, and G. Röpke, J. Low Temp. Phys. 189, 133 (2017).

[38] G. Röpke, A. Schnell, P. Schuck, and P. Nozieres, Phys. Rev. Lett. 80, 3177 (1998).

[39] M. Beyer, S. A. Sofianos, C. Kuhrts, G. Röpke, and P. Schuck, Phys. Lett. B 488, 247 (2000).

[40] S. Typel, G. Röpke, T. Klähn, D. Blaschke, and H. H. Wolter, Phys. Rev. C 81, 015803 (2010).

[41] H. Tajima and S. Uchino, New J. Phys. 20, 073048 (2018).

[42] Lai Hnin Phyu, H. Moriya, W. Horiuchi, K. Iida, K. Noda, and M. T. Yamashita, Prog. Theor. Exp. Phys. 2020, 093 D01 (2020). 